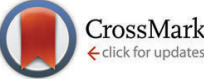

Cite this: Phys. Chem. Chem. Phys., 2014, 16, 26137

Received 22nd September 2014, Accepted 22nd October 2014

DOI: $10.1039 / c 4 c p 04261 a$

www.rsc.org/pccp

\title{
Fronts and patterns in a spatially forced CDIMA reaction
}

\author{
Lev Haim, ${ }^{\text {ab }}$ Aric Hagberg, ${ }^{* c}$ Raphael Nagao, ${ }^{\text {de }}$ Asher Preska Steinberg, ${ }^{\dagger}$ \\ Milos Dolnik, Irving R. Epstein ${ }^{d}$ and Ehud Meron ${ }^{\text {ag }}$
}

\begin{abstract}
We use the CDIMA chemical reaction and the Lengyel-Epstein model of this reaction to study resonant responses of a pattern-forming system to time-independent spatial periodic forcing. We focus on the $2: 1$ resonance, where the wavenumber of a one-dimensional periodic forcing is about twice the wavenumber of the natural stripe pattern that the unforced system tends to form. Within this resonance, we study transverse fronts that shift the phase of resonant stripe patterns by $\pi$. We identify phase fronts that shift the phase discontinuously, and pairs of phase fronts that shift the phase continuously, clockwise and anti-clockwise. We further identify a front bifurcation that destabilizes the discontinuous front and leads to a pair of continuous fronts. This bifurcation is the spatial counterpart of the nonequilibrium Ising-Bloch (NIB) bifurcation in temporally forced oscillatory systems. The spatial NIB bifurcation that we find occurs as the forcing strength is increased, unlike earlier studies of the NIB bifurcation. Furthermore, the bifurcation is subcritical, implying a range of forcing strength where both discontinuous Ising fronts and continuous Bloch fronts are stable. Finally, we find that both Ising fronts and Bloch fronts can form discrete families of bound pairs, and we relate arrays of these front pairs to extended rectangular and oblique patterns
\end{abstract}

\section{Introduction}

A canonical experimental model for studying stationary patterns far from equilibrium is the chlorite-iodide-malonic acid (CIMA) reaction. ${ }^{1}$ This model reaction was used to verify, for the first time, the prediction of Alan Turing ${ }^{2}$ of a diffusion-induced patternforming instability, ${ }^{3}$ and it has served as a major experimental tool for studying pattern-formation phenomena in reaction-diffusion systems ever since. ${ }^{4}$ A modified version of that reaction, the chlorine dioxide-iodine-malonic acid (CDIMA) reaction, ${ }^{5}$ has been used to study pattern-formation effects induced by periodic forcing. The CDIMA reaction is photosensitive and can be forced in time or in space by periodic illumination with white light. Periodic forcing of this kind has been studied as a means of controlling patterns.

\footnotetext{
${ }^{a}$ Physics Department, Ben-Gurion University of the Negev, Beer-Sheva 84105, Israel

${ }^{b}$ Department of Oncology, Soroka University Medical Center, Beer-Sheva, 84101, Israel

${ }^{c}$ Center for Nonlinear Studies, Theoretical Division, Los Alamos National Laboratory, Los Alamos, New Mexico 87545, USA

${ }^{d}$ Department of Chemistry and Volen Center for Complex Systems, MS 015, Brandeis University, Waltham, MA 02454, USA

${ }^{e}$ Department of Chemistry, Saint Louis University, St. Louis, MO 63103, USA

${ }^{f}$ Division of Chemistry and Chemical Engineering, California Institute of Technology, Pasadena, CA 91125, USA

${ }^{g}$ Department of Solar Energy and Environmental Physics, BIDR,

Ben-Gurion University of the Negev, Sede Boqer Campus, 84990, Israel
}

Periodic forcing can be used to lock and control the frequency of periodic oscillations ${ }^{6-9}$ or the wavenumber of a periodic pattern, ${ }^{10-14}$ to stabilize and enhance patterns, ${ }^{15}$ or to induce new controllable patterns. ${ }^{6,10,11,15-19}$

There are two main mechanisms by which periodic forcing can induce new patterns. The first is a new pattern-forming instability of the original uniform state. In the case of temporal forcing of an oscillatory system the forcing can induce a finitewavenumber Turing-like instability. ${ }^{8,17}$ In the case of spatial forcing of a pattern-forming system the forcing can induce rectangular and oblique patterns. ${ }^{18}$ The second mechanism is multiplicity of stable phase states. The unforced system has a continuous translational symmetry, in time for an oscillatory system and in space for a pattern-forming system, which results in a continuous family of periodic solutions (phase states) whose phases span the whole circle. The forcing breaks this continuous symmetry, leaving a discrete set of stable phase states. Multistability of this kind allows for patterns comprising alternating domains of different phase states. ${ }^{9,16,20,21}$

We restrict our attention here to pattern formation phenomena associated with bistability of two phase states in spatially forced pattern-forming systems. The two phase states describe periodic stripe patterns with a phase shift of $\pi$ with respect to one another. Bistability of this kind can be achieved when the wavenumber of a one-dimensional (1d) periodic forcing is about twice the wavenumber of the natural stripe pattern that 
the unforced system tends to form. A characteristic feature of bistable systems in general is the possible existence of stable front structures that are biasymptotic to the two stable states. In the present context, the fronts form localized transition zones that shift the pattern phase by $\pi$. Because of the anisotropy of stripe patterns, there are two types of such fronts: longitudinal fronts that are parallel to the stripes, and transverse fronts that are perpendicular to the stripes. Studies of a simple pattern formation model - the Swift-Hohenberg equation - have uncovered two forms of each front type; a front that shifts the phase discontinuously and a front that shifts the phase gradually and smoothly. ${ }^{22}$ These studies have also identified longitudinal and transverse front bifurcations, as the forcing strength is decreased, which destabilize fronts that shift the phase discontinuously and give rise to pairs of fronts that shift the phase continuously, either clockwise or anticlockwise. These front bifurcations are the spatial counterparts of the so-called nonequilibrium Ising-Bloch (NIB) bifurcation in temporally forced oscillatory systems. ${ }^{23-26}$ We refer to fronts that shift the phase discontinuously and continuously as Ising and Bloch fronts, respectively.

In this paper we study the spatial NIB bifurcation for transverse fronts in a spatially forced CDIMA reaction and in the LengyelEpstein (LE) model that describes this reaction. ${ }^{27}$ We further use the LE model to study numerically the interactions between pairs of Ising and Bloch fronts and relate the results of this study to the existence of rectangular and oblique patterns.

\section{Experimental setup for the spatially forced CDIMA reaction}

The patterns were studied in a continuously-fed, unstirred, onesided reactor (CFUR). The reactor consisted of a $0.3 \mathrm{~mm}$ thick $2 \%$ agarose (Fluka) gel layer with diameter of working area $25 \mathrm{~mm}$ placed between a glass window and a continuously-fed stirred tank reactor (CSTR), which served as a feeding chamber. Between the CFUR and the CSTR were two membranes: a cellulose nitrate membrane (Whatman, pore size $0.45 \mu \mathrm{m}$, thickness $0.12 \mathrm{~mm}$ ) beneath the gel for enhanced contrast, and, to provide rigidity to the gel and to separate it from the stirred feeding chamber, an anopore membrane (Whatman, pore size $0.2 \mu \mathrm{m}$, impregnated with $4 \%$ agarose gel, overall thickness $0.10 \mathrm{~mm}$ ) placed underneath the cellulose nitrate membrane. The CSTR was fed with three reagent solutions: (i) $I_{2}$ (Aldrich), (ii) a mixture of malonic acid (MA, Aldrich) and poly-(vinyl alcohol) (PVA, Aldrich, average molecular weight 9000-10 000); and (iii) $\mathrm{ClO}_{2}$ prepared as described in ref. 28. The PVA is a binding agent for triiodide ions and acts as a color indicator.

The initial concentrations of reagents fed into the CSTR were the same in all experiments: $\left[\mathrm{I}_{2}\right]=0.4 \mathrm{mM},[\mathrm{MA}]=2.1 \mathrm{mM}$, $\left[\mathrm{ClO}_{2}\right]=0.14 \mathrm{mM}$, and $[\mathrm{PVA}]=10 \mathrm{~g} \mathrm{~L}^{-1}$. Each of the input solutions contained $10 \mathrm{mM}$ sulfuric acid (Fisher). The residence time of the reagents in the CSTR was $160 \mathrm{~s}$. The temperature was $4.0 \pm 0.2{ }^{\circ} \mathrm{C}$. With these conditions, a labyrinthine pattern develops spontaneously. A computer-controlled DLP projector (Dell 1510X) was used to implement the uniform and spatially periodic white light illumination of the CFUR. The light intensity was measured with a Newport 1815 optical power meter. A CCD Pixelink camera was used to record images of the patterns. Snapshots were taken in ambient light of $0.6 \mathrm{~mW} \mathrm{~cm}^{-2}$ with no illumination projected on the CFUR.

\section{Mathematical model of the spatially forced CDIMA reaction}

We studied the modified Lengyel-Epstein (LE) model $^{29}$ that takes the effect of illumination into account:

$$
\begin{aligned}
& u_{t}=a-u-\frac{4 u v}{1+u^{2}}-w(x)+\nabla^{2} u, \\
& v_{t}=\sigma\left[b\left(u-\frac{u v}{1+u^{2}}+w(x)\right)+d \nabla^{2} v\right] .
\end{aligned}
$$

Here $u$ and $v$ are dimensionless concentrations of iodide and chlorite ions, respectively; $a, b, d, \sigma$ are dimensionless parameters, and $w(x)$ denotes the rate of the photochemical reaction. In the present study, $w$ is a one-dimensional periodic function of the spatial $x$-coordinate and is independent of the $y$-coordinate.

In the experiments described in Section 2, we used a spatial square-wave forcing. This forcing form can be captured in the model by the function

$$
w(x)=w_{0}+\frac{\gamma}{2}\left(1+\operatorname{sign}\left[\cos \left(k_{\mathrm{f}} x\right)\right]\right),
$$

where $k_{\mathrm{f}}$ is the forcing wavevector, $\gamma$ is the forcing strength and $w_{0}$ represents the ambient light. This form can be expanded as a Fourier cosine series,

$$
w(x)=w_{0}+\frac{\gamma}{2}+\sum_{n=1}^{\infty} \gamma_{n} \cos \left(n k_{\mathrm{f}} x\right),
$$

where

$$
\gamma_{n}=\frac{2 \gamma}{n \pi} \sin \left(\frac{n \pi}{2}\right)
$$

In the following, we will approximate $w$ by considering only the first and largest term in the expansion, $(n=1)$ :

$$
w(x)=w_{0}+\frac{\gamma}{2}+\frac{2 \gamma}{\pi} \cos \left(k_{\mathrm{f}} x\right) .
$$

We have checked that taking instead the full expansion, or the square wave form, does not have any qualitative effect on the results, and the quantitative effect is insignificant.

The LE model (1) with no forcing $(\gamma=0)$ has the stationary uniform solution

$$
u_{0}=\frac{a}{5}-w_{0}, \quad v_{0}=\left(1+u_{0}^{2}\right)\left(\frac{w_{0}}{u_{0}}+1\right) .
$$

This solution goes through a Turing bifurcation to stationary periodic patterns as $b$ is decreased below a critical value

$$
b_{\mathrm{T}}=\frac{u_{0} d k_{\mathrm{T}}^{4}}{5\left(1+u_{0}^{2}\right)},
$$


where

$$
k_{\mathrm{T}}^{2}=-5+2 \sqrt{5} \sqrt{\frac{\left(u_{0}^{2}-1\right) v_{0}}{\left(u_{0}^{2}+1\right)^{2}}+1},
$$

and $k_{\mathrm{T}}$ is the Turing wavenumber. The stationary uniform solution also goes through a Hopf bifurcation to uniform oscillations as $b$ is decreased below another critical value $b_{\mathrm{H}}=$ $k_{\mathrm{T}}^{2}\left(k_{\mathrm{T}}^{2}+10\right)\left(1+u_{0}^{2}\right) / 5 \sigma u_{0}$. In all studies that follow, we chose values of $a, \sigma, d$ so that $b_{\mathrm{H}}<b_{\mathrm{T}}$. This guarantees that the Turing instability is the first to be encountered as $b$ is decreased. We focus on the 2:1 resonance, for which $k_{\mathrm{f}} \approx 2 k_{\mathrm{T}}$. Since the experiments are limited to $k_{\mathrm{f}}<2 k_{\mathrm{T}}$, we restrict ourselves to this range also in the model studies. The range $k_{\mathrm{f}}>2 k_{\mathrm{T}}$ is interesting because rectangular and oblique patterns are ruled out. ${ }^{18}$ This case will be considered in a future study.

\section{Bistability of fronts in the CDIMA reaction}

\subsection{Data analysis}

Ising and Bloch fronts are localized structures that separate one resonant stripe pattern from a symmetric stripe pattern whose phase is shifted by $\pi$. Assuming a forcing wavevector $k_{\mathrm{f}}=k_{\mathrm{f}} \hat{\mathbf{x}}$, where $\mathbf{k}_{\mathrm{f}}$ is sufficiently close to $2 k_{\mathrm{T}}$, the common wavevector $\mathbf{k}=$ $k \hat{\mathbf{x}}$ of the two symmetric stripe patterns is locked to the forcing wavevector in a $2: 1$ resonance, $\left(k_{\mathrm{f}}: k\right)=(2: 1)$. This resonant response occurs in a $k_{\mathrm{f}}$ range whose size depends on the forcing strength. A stripe pattern that contains an Ising or Bloch front can be approximated as

$$
u(x, y) \approx u_{0}+A(x, y) \mathrm{e}^{i k_{\mathrm{f} x} / 2}+\text { c.c. }+\ldots,
$$

where c.c. is the complex conjugate, the ellipses denote higher order harmonic contributions, and $u(x, y)$ represents the experimental data, or the numerically computed $u$ field in the LE model (a similar expression holds for the $v$ field in the LE model with the amplitude factor $A$ replaced by $c A$, where $c$ is a constant). Longitudinal fronts are captured by amplitudes $A(x)$ that are independent of $y$, while transverse fronts are captured by amplitudes $A(y)$ that are independent of $x$. We note that, since the system sizes (both in the experiment and model) are much larger than both the pattern wavelengths and the front widths, any boundary effects are negligible.

Transverse Ising fronts can be distinguished from transverse Bloch fronts by plotting phase trajectories in the complex- $A$ plane that are parameterized by $y .^{9,16,23}$ Trajectories that go through the origin $(A=0)$ describe Ising fronts, $\dagger$ whereas trajectories that bypass the origin describe Bloch fronts. To obtain the complex valued amplitude $A$, we project $u(x, y)$ onto $\exp \left(i k_{\mathrm{f}} x / 2\right)$ :

$$
A(y)=\frac{1}{L} \int_{0}^{L} u(x, y) \mathrm{e}^{-i k_{\mathrm{f}} x / 2} \mathrm{~d} x,
$$

$\dagger$ Since Ising fronts involve a discontinuous phase jump, the modulus $|A|$ must vanish to avoid a singularity in $A$. where $L$ is the system size, chosen to be an integer multiple of the pattern's wavelength.

\subsection{Experimental results}

We studied transverse Ising and Bloch fronts, varying the forcing strength, starting both from Ising-front and Bloch-front initial conditions. Initial conditions in the experiments were prepared as follows. After a labyrinthine pattern of the unforced reaction becomes stationary, the system is brought to a spatially uniform steady state using homogeneous illumination for 2 min with intensity $100 \mathrm{~mW} \mathrm{~cm}^{-2}$. Then a mask is placed between the source light and the reactor, and the image of the mask is focused on the surface of the gel. The illumination through the mask is applied for $60 \mathrm{~min}$, and the maximum light intensity that reaches the gel is chosen to be $6.0 \mathrm{~mW} \mathrm{~cm}^{-2}$. The mask consists of two patterns with a phase shift of $\pi$, as Fig. 1 shows. Both patterns have wavenumbers $k_{\mathrm{f}}$ equal to the intrinsic (natural) wavenumber $k_{\mathrm{T}}$ of the labyrinthine Turing patterns that form in the absence of illumination. Two types of this mask are used, one that mimics an Ising front (left panel in Fig. 1) and one that mimics a Bloch front (right panel in Fig. 1). These mask types create initial conditions that result in transverse Ising and Bloch fronts, respectively, when the $2: 1$ forcing is applied. The $2: 1$ forcing is achieved by illuminating the gel for $60 \mathrm{~min}$ through a second mask (not shown) with wavenumber twice as large, $k_{\mathrm{f}}=2 k_{\mathrm{T}}$, and aligned with the patterns from the first mask. The forcing strength is varied by changing the maximum intensity of transmitted light.

Fig. 2 and 3 show examples of transverse Ising and Bloch fronts observed in the CDIMA reaction after the second mask

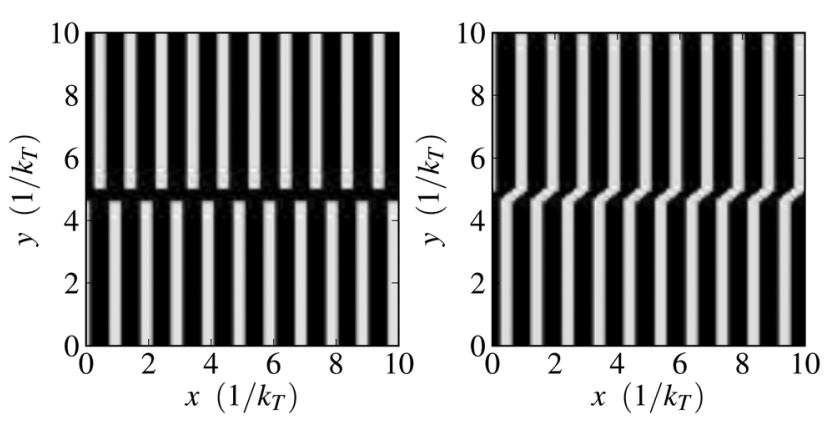

Fig. 1 Masks used to create transverse Ising (left) and Bloch (right) fronts. The mask shown is 10 wavelengths with wavelength $\lambda=0.37 \mathrm{~mm}$.
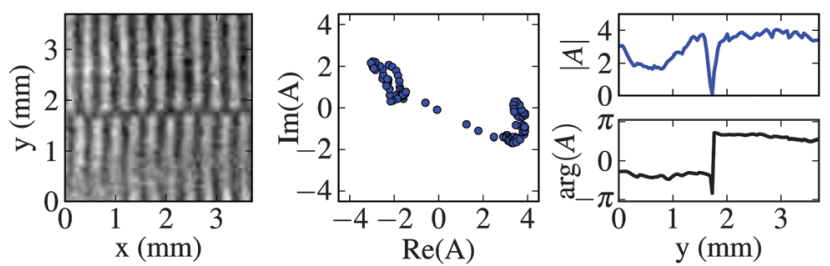

Fig. 2 An observed transverse Ising front in the CDIMA reaction with forcing intensity $I=28 \mathrm{~mW} \mathrm{~cm}{ }^{-2}$. The phase trajectory in the complex amplitude plane goes through the origin (middle panel) and the modulus of the amplitude vanishes there (right panel). The front shifts the pattern's phase discontinuously by $\pi$ (right panel). 

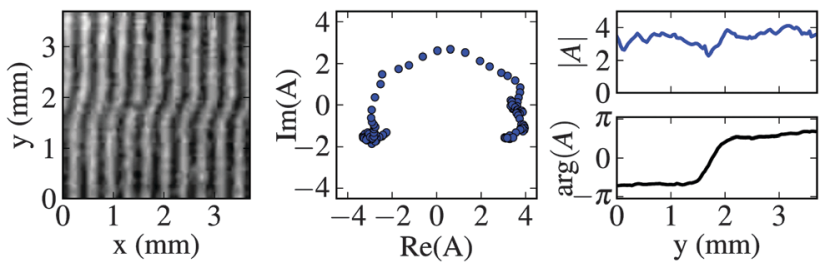

Fig. 3 An observed transverse Bloch front in the CDIMA reaction with forcing intensity $I=28 \mathrm{~mW} \mathrm{~cm}{ }^{-2}$. The phase trajectory in the complex amplitude plane bypasses the origin (middle panel) and the modulus of the amplitude is bounded away from zero (right panel). The front shifts the pattern's phase continuously by $\pi$ (right panel).

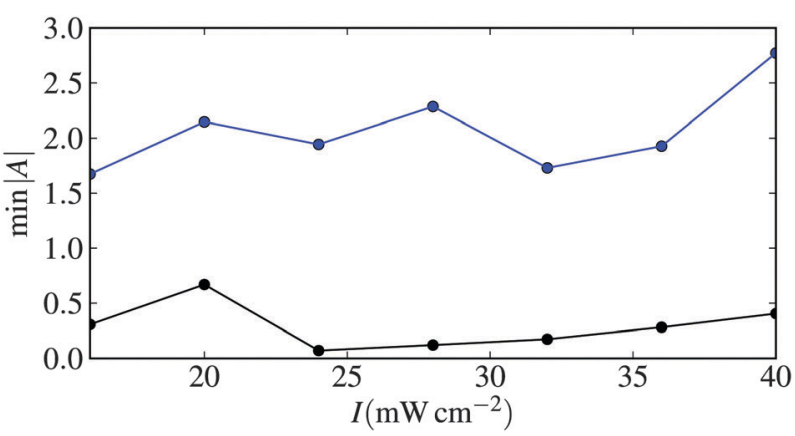

Fig. 4 Bistability of Ising and Bloch fronts in the CDIMA reaction. The blue (upper) dots represent Bloch fronts while the black (lower) points represent Ising fronts.

has been used. While in Fig. $3|A|$ is bounded away from zero, indicating a Bloch front, in Fig. 2 it approaches zero at a point (within the limited experimental resolution), indicating an Ising front. The smooth phase change across the Bloch fronts vs. the phase jump across the Ising front (which necessitates a zero of the amplitude) is also seen in the patterns themselves.

Varying the forcing strength up and down, starting both with Ising and Bloch fronts as initial conditions, we found that both fronts are stable in the experimentally realizable forcing range, as Fig. 4 shows. The bistability of Ising and Bloch fronts allows for mixed-front patterns in which the transverse front that separates the $\pi$-shifted patterns consists of both Ising parts and Bloch parts.

\section{A subcritical front bifurcation in the LE model}

We also found transverse Ising and Bloch fronts in numerical studies of the LE model (eqn (1) and (2)), in a parameter range that corresponds to the reaction conditions, as Fig. 5 and 6 show. By scanning a wide enough range of forcing strengths, we were able to observe a NIB bifurcation. As Fig. 8 shows, the bifurcation is subcritical, implying a bistability range of Ising and Bloch fronts, as found in the experiment. Note that Ising fronts destabilize to Bloch fronts as the forcing strength is increased, unlike earlier studies of the NIB bifurcation, where Bloch fronts appear as the forcing strength is decreased. ${ }^{17}$
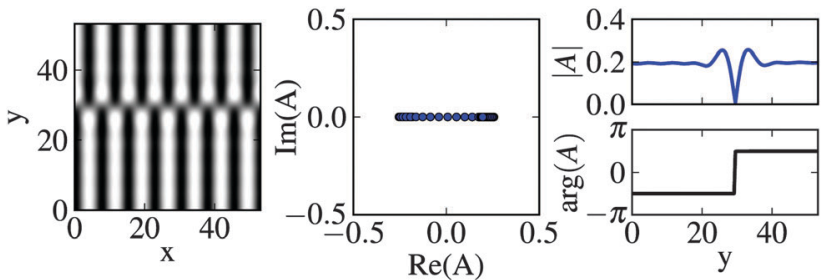

Fig. 5 A transverse Ising front in the $v$ field of the LE model. The phase trajectory in the complex amplitude plane goes through the origin (middle panel), and the modulus of the amplitude vanishes there (right panel). Parameters: $a=12, d=1, \sigma=50, w_{0}=0, \gamma=0.6, k_{\mathrm{f}}=1.8 k_{\mathrm{T}}$, and $b=0.38$.
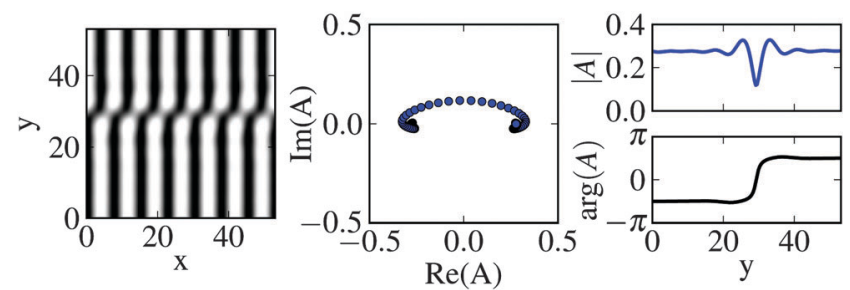

Fig. 6 A transverse Bloch front in the $v$ field of the LE model. The phase trajectory in the complex amplitude plane bypasses the origin (middle panel), and the modulus of the amplitude is bounded away from zero (right panel). Parameters: $a=12, d=1, \sigma=50, w_{0}=0, \gamma=0.6, k_{f}=1.8 k_{T}$ and $b=0.35$.

The reverse nature of the spatial NIB bifurcation in the LE model can be understood, at least partly, by deriving an amplitude equation for stripe patterns in the LE model (assuming an infinite system). Approximating a solution of the LE model as in (6), the amplitude $A$ satisfies the equation

$$
\tau \partial_{t} A=\mu A-\eta|A|^{2} A+\Gamma A^{\star}+\left[\left(\partial_{x}-i \nu\right)-i \frac{\partial_{y}^{2}}{2 k_{\mathrm{T}}}\right]^{2} A,
$$

where $A^{*}$ is the complex conjugate of $A, \mu$ represents the distance from the Turing bifurcation, $\nu=k_{\mathrm{T}}-k_{\mathrm{f}} / 2$ is the wavenumber detuning from exact $2: 1$ resonance, and $\Gamma$ is a function of the forcing strength $\gamma$. The explicit forms of these parameters, as well as those of $\alpha, \tau, \eta$, are given elsewhere. ${ }^{30}$ This equation cannot describe the observed patterns in the CDIMA reaction in any quantitative manner, because the reaction has been conducted far from the onset of the Turing instability. It does show, however, that the coefficient $\Gamma$ of the forcing term $A^{*}$, which in simple systems is proportional to the forcing strength $\gamma$, depends non-monotonically on $\gamma$ as shown in Fig. 7 . This implies the possible existence of forcing intensities in which the effect of the forcing on the chemical reaction decreases upon strengthening the forcing, which may explain the reverse nature of the NIB bifurcation.

\section{Front pairs as building blocks for extended patterns}

The periodic spatial forcing can induce extended rectangular and oblique patterns, as shown in the simulations of the LE model presented in Fig. 9. The power spectra of these patterns 


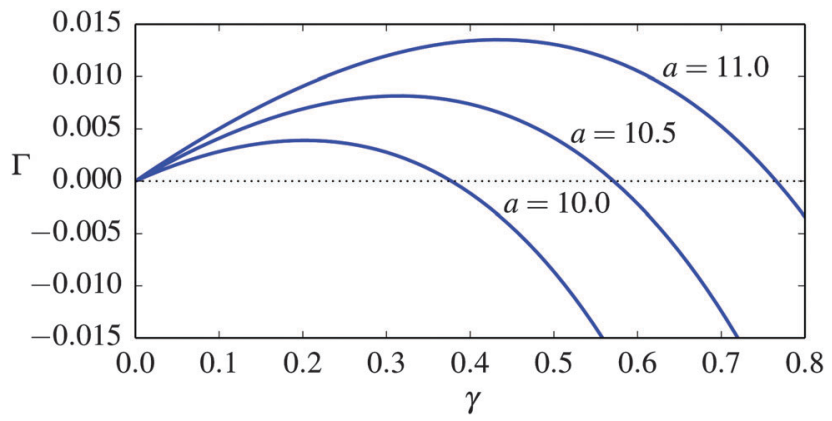

Fig. 7 The dependence of the coefficient $\Gamma$ in eqn (8) on the forcing strength, $\gamma$, for different values of the parameter $a$ and for $k_{\mathrm{f}}=1.8 \mathrm{k}_{\mathrm{T}}(\Gamma$ is independent of $d$ and $\sigma$ ).

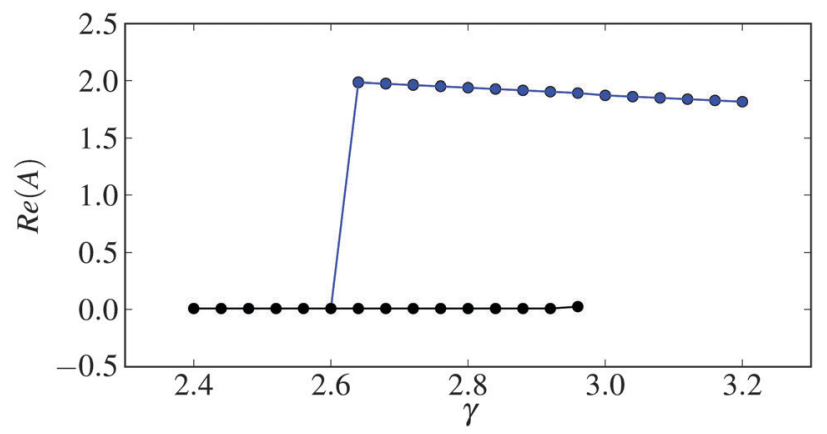

Fig. 8 NIB bifurcation in the LE model (1). The blue (upper) dots represent Bloch fronts while the black (lower) points represent Ising fronts. Parameters: $a=30, d=1, \sigma=100, w_{0}=0, b=1.7$ and $k_{\mathrm{f}}=1.8 \mathrm{kT}_{\mathrm{T}}$.
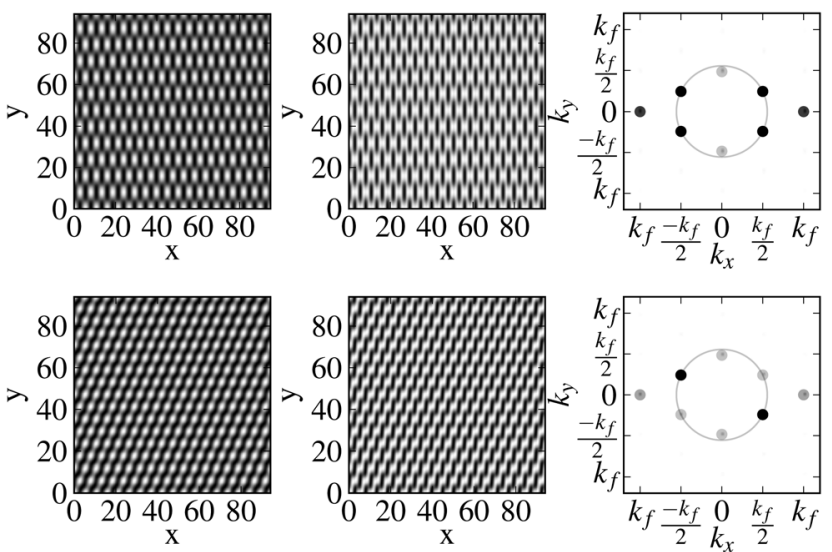

Fig. 9 Resonant 2:1 rectangular pattern (top row) and oblique pattern (bottom row) in the LE model. The left and middle frames show the $v$ and $u$-field patterns in the $(x, y)$ plane. The right frames show the corresponding spatial Fourier transform of $u$ in the $\left(k_{x}, k_{y}\right)$ plane. The shading of the circles in the Fourier plane indicates the strength of the modes (darker is higher). Note the asymmetry in the strength of the two oblique modes for the oblique pattern and the presence of the forcing wave-vector in the rectangular pattern. Parameters: $a=12, d=1, \sigma=50, w_{0}=0, \gamma=0.6$, $k_{\mathrm{f}}=1.8 k_{\mathrm{T}}$, and $b=0.38$ (rectangular pattern) and $b=0.35$ (oblique pattern).

show the presence of the oblique modes, $\exp \left(i k_{x} x \pm i k_{y} y\right)$, and the wave-vector components $k_{x}=k_{\mathrm{f}} / 2$ and $k_{y}$ satisfying $k_{x}^{2}+k_{y}^{2}=$ $k_{\mathrm{T}}^{2}$ indicate that these patterns are resonant. ${ }^{31}$ Note that the

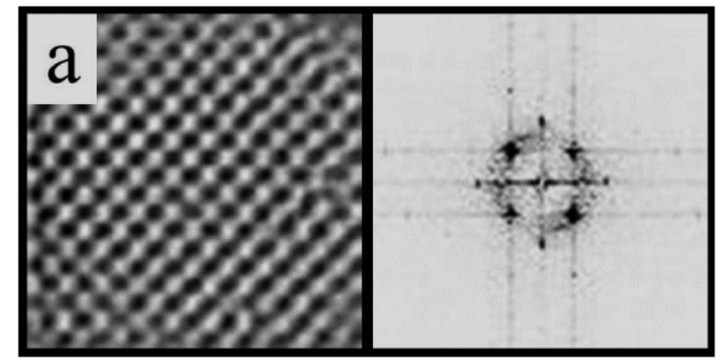

Fig. 10 Resonant 2:1 rectangular pattern in the spatially forced CDIMA reaction (left) and the corresponding $2 \mathrm{~d}$ Fourier plane, which shows the strong presence of two oblique modes (right). Forcing parameters: $k_{\mathrm{f}}=$ $1.67 \mathrm{k}_{\mathrm{T}}, I_{\max }=7.62$. Snapshots were taken $2 \mathrm{~h}$ after the start of forcing.

difference between rectangular and oblique patterns lies in the relative weights of the two oblique modes: equal (unequal) absolute values of their amplitudes imply rectangular (oblique) modes. We found extended resonant rectangular patterns in the CDIMA reaction, too, as Fig. 10 shows. Note the strong presence of a resonant stripe mode with wave-vector $k_{\mathrm{f}} \hat{\mathbf{x}}$, and its complex conjugate mode at $-k_{\mathrm{f}} \hat{\mathbf{x}}$, in the power spectrum of the rectangular pattern, which imparts to this pattern a rhombic form. This can be attributed to the resonance condition between this stripe mode and the two oblique modes, $\mathbf{k}_{+}+\mathbf{k}_{-}+k_{\mathrm{f}} \hat{\mathbf{x}}=0$, where $\mathbf{k}_{ \pm}=-\frac{k_{\mathrm{f}}}{2} \hat{\mathbf{x}} \mp k_{y} \hat{\mathbf{y}}$.

The question we now address, using the LE model, is whether there exists a relation between the extended rectangular and oblique patterns and the localized Ising and Bloch fronts. The motivation for this question is mainly visual - rectangular patterns appear similar to arrays of Ising-front pairs, and oblique patterns appear similar to arrays of Bloch-front pairs. The existence of two types of Bloch fronts is consistent with the existence of two types of oblique patterns. In order for an array of front pairs to form a stable extended pattern, the front interactions cannot be attractive at any inter-front distance. We therefore used the LE model to study the interactions between pairs of Ising fronts and pairs of Bloch fronts in the range $k_{\mathrm{f}}<2 k_{\mathrm{T}}$ where rectangular and oblique patterns exist. Fig. 11 shows plots of the asymptotic distance $d_{\mathrm{f}}$ between a pair of Ising fronts and the initial distance (upper left panel) and similar plots for pairs of Bloch fronts (lower left panel). At longer initial distances the fronts are too far to interact significantly, and the final distance equals the initial one. As the initial distance is decreased, a step-like graph appears for both Ising and Bloch fronts, indicating the existence of discrete solution families describing stable bound front pairs with decreasing distances down to a typical minimal distance.

To test whether extended rectangular and oblique patterns can be viewed, respectively, as arrays of Ising and Bloch front pairs, we superimposed bound pair front solutions on the extended solutions. As Fig. 12 shows, the spatial profile in the $y$ direction of the shortest-distance Ising-front pair nicely overlaps the rectangular pattern and, similarly, the shortestdistance Bloch-front pair overlaps the oblique pattern. These results support the view of Ising-front bound pairs and Blochfront bound pairs as building blocks of rectangular and oblique patterns, respectively, provided that we focus on the shortestdistance bound pairs. 

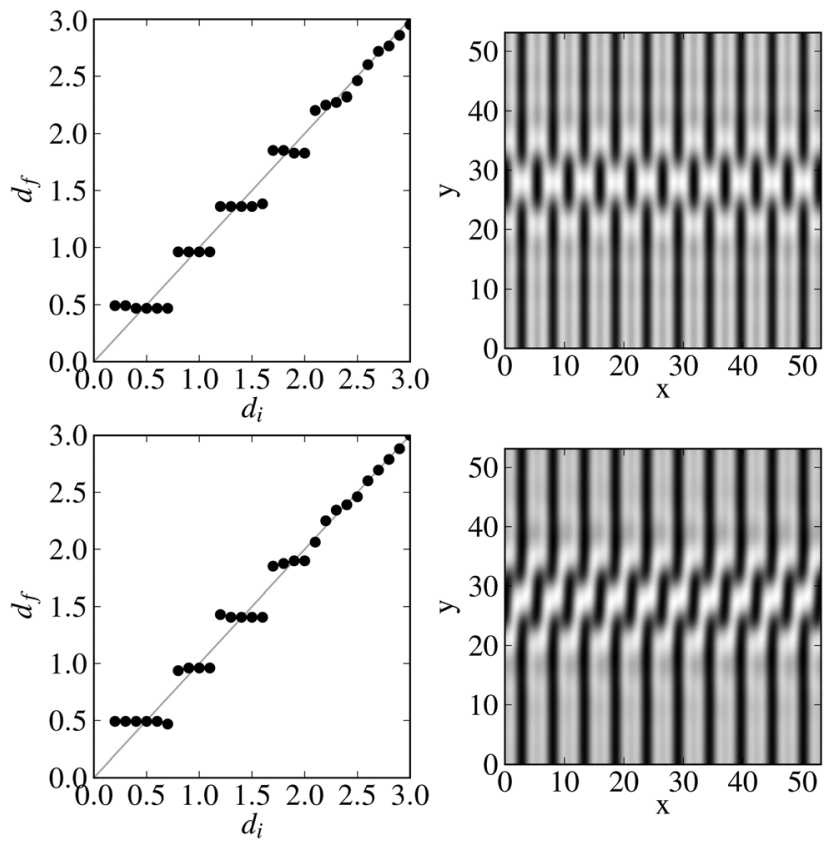

Fig. 11 Numerical solutions showing the asymptotic distance $d_{\mathrm{f}}$ between a pair of Ising fronts vs. the initial distance (upper left panel, $b=0.35$ ) and a similar plot for pairs of Bloch fronts (lower left panel, $b=0.38$ ). The staircase forms describe bound front-pair solutions at increasing distances. The right panels show the corresponding shortest-distance front pairs. Parameters: $a=12, d=1, \sigma=50, w_{0}=0, k_{\mathrm{f}}=1.8 \mathrm{kT}_{\mathrm{T}}$ and $\gamma=0.6$.
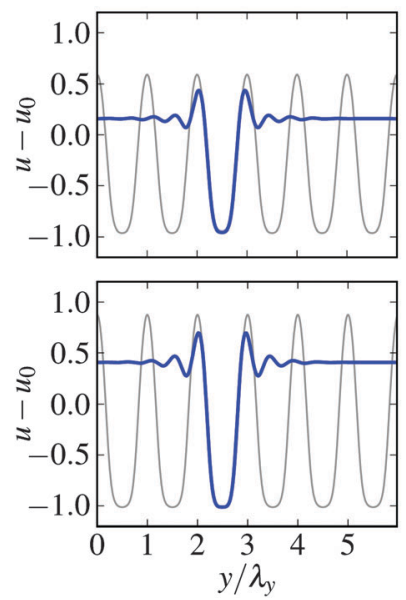
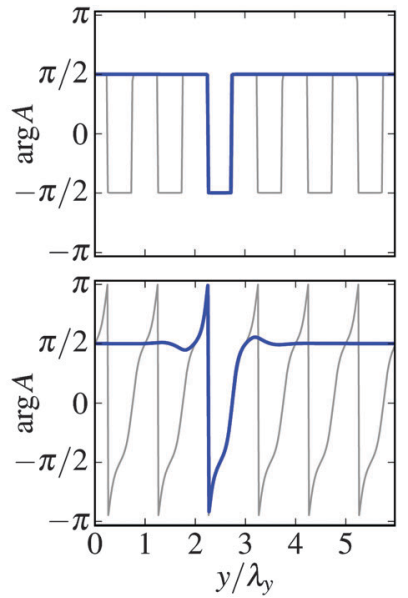

Fig. 12 Spatial profiles of $u-u_{0}$ (left panels) and $\arg A$ (right panels), evaluated at $x=0$ as functions of $y$ by solving (1) and using (7). The upper (lower) panel corresponds to a bound Ising (Bloch) pair denoted by a thick black color superimposed on a rectangular (oblique) pattern denoted by thin gray color Parameters: $\lambda_{y}=2 \pi /\left(k_{\mathrm{T}}{ }^{2}-k_{\mathrm{f}}{ }^{2} / 4\right)^{-1 / 2}, a=12, d=1, \sigma=50, w_{0}=0, k_{\mathrm{f}}=1.8 k_{\mathrm{T}}$, $\gamma=0.6$ with $b=0.38$ for lower panels and $b=0.35$ for upper panels.

\section{Conclusions}

We studied here the NIB bifurcation for transverse fronts using a spatially forced CDIMA reaction and the Lengyel-Epstein (LE) model. We demonstrated the existence of a spatial NIB bifurcation and showed that it is a subcritical bifurcation, implying bistability of stable transverse Ising and transverse Bloch fronts. Subcritical NIB bifurcations have been found earlier in the context of temporally forced oscillations. ${ }^{32,33}$ Unlike earlier studies of the NIB bifurcation, both in temporal and spatial contexts, Ising fronts here were found to lose stability to Bloch fronts as the forcing strength is increased rather than decreased. We also studied the interactions between pairs of Ising fronts and between pairs of Bloch fronts and found discrete families of bound Ising-front pairs and of bound Bloch-front pairs ${ }^{22}$ with increasing inter-front distance. We further showed that rectangular patterns can be viewed as arrays of Ising-front bound pairs and oblique patterns as arrays of Bloch-front bound pairs, where in both cases the bound pairs are the shortest-distance ones. These findings connect the two distinct mechanisms by which periodic spatial forcing induces new patterns: a nonuniform instability of a uniform state to rectangular or oblique patterns, and bistability of phase states and patterns consisting of front pairs as building blocks, i.e., patterns of alternating phase states.

The results described above imply the existence of a forcing range where three different $1 \mathrm{~d}$ front solutions, all shifting the pattern phase by $\pi$, are stable: an Ising front and a pair of Bloch fronts. Multiplicity of stable $1 \mathrm{~d}$ front solutions allows for $2 \mathrm{~d}$ localized structures, as front lines that shift the phase by $\pi$ can consist of different front solutions with an intervening transition zone that forms the $2 \mathrm{~d}$ localized structure. In temporally forced oscillatory systems transition zones of this kind between counter-propagating Bloch fronts form spiral vortices. ${ }^{34}$ In spatially forced pattern forming systems, such transitions zones form dislocation-type defects. The availability of a stable Ising front allows for an additional type of $2 \mathrm{~d}$ localized structure - a structure that that forms a transition zone between the Ising front and one of the Bloch fronts. Structures of this kind and additional structures have been found both in the experiments and in the model simulations and call for further studies.

The CDIMA reaction may be a good candidate for studying dual-mode fronts. In the absence of fronts, decreasing $b$ below the Hopf bifurcation threshold, $b_{\mathrm{H}}$, may not give rise to oscillations. This is because the large amplitude Turing mode is likely to damp the small amplitude Hopf mode through nonlinear coupling. However, in the presence of an Ising front, where the amplitude $A$ vanishes, the nonlinear damping may be too weak to prevent the local growth of the Hopf mode. Such a growth should lead to oscillating Ising fronts. ${ }^{35,36}$

\section{Acknowledgements}

The support of the United States-Israel Binational Science Foundation (Grant No. 2008241) is gratefully acknowledged. Part of this work was funded by the Laboratory Directed Research and Development program at Los Alamos National Laboratory under Department of Energy Contract No. DE-AC52-06NA25396.

\section{References}

1 P. D. Kepper, I. R. Epstein, M. Orbán and K. Kustin, J. Phys. Chem., 1982, 86, 170-171. 
2 A. Turing, Philos. Trans. R. Soc. London, Ser. B, 1952, 237, 37-72.

3 V. Castets, E. Dulos, J. Boissonade and P. De Kepper, Phys. Rev. Lett., 1990, 64, 2953-2956.

4 I. R. Epstein and J. A. Pojman, Introduction to Nonlinear Chemical Dynamics. Oscillations, Waves, Patterns and Chaos, Oxford University Press, New York, 1998.

5 I. Lengyel, G. Rábai and I. R. Epstein, J. Am. Chem. Soc., 1990, 112, 4606-4607.

6 H.-K. Park, Phys. Rev. Lett., 2001, 86, 1130-1133.

7 A. Yochelis, C. Elphick, A. Hagberg and E. Meron, Physica D, 2004, 199, 201-222.

8 A. Yochelis, C. Elphick, A. Hagberg and E. Meron, Europhys. Lett., 2005, 69, 170.

9 A. L. Lin, A. Hagberg, E. Meron and H. L. Swinney, Phys. Rev. E: Stat., Nonlinear, Soft Matter Phys., 2004, 69, 066217.

10 Y. Mau, A. Hagberg and E. Meron, Phys. Rev. Lett., 2012, 109, 034102.

11 M. Dolnik, T. Bánsági, S. Ansari, I. Valent and I. R. Epstein, Phys. Chem. Chem. Phys., 2011, 13, 12578-12583.

12 D. Feldman, R. Nagao, T. Bansagi Jr., I. R. Epstein and M. Dolnik, Phys. Chem. Chem. Phys., 2012, 14, 6577-6583.

13 Y. Mau, L. Haim, A. Hagberg and E. Meron, Phys. Rev. E: Stat., Nonlinear, Soft Matter Phys., 2013, 88, 032917.

14 L. Haim, Y. Mau and E. Meron, Phys. Rev. E: Stat., Nonlinear, Soft Matter Phys., 2014, 90, 022904.

15 R. Manor, A. Hagberg and E. Meron, New J. Phys., 2009, 11, 063016.

16 A. L. Lin, M. Bertram, K. Martinez, H. L. Swinney, A. Ardelea and G. F. Carey, et al., Phys. Rev. Lett., 2000, 84, 4240-4243.

17 A. Yochelis, A. Hagberg, E. Meron, A. L. Lin and H. L. Swinney, SIAM J. Appl. Dyn. Syst., 2002, 1, 236-247.

18 R. Manor, A. Hagberg and E. Meron, EPL, 2008, 83, 10005.

19 G. Freund and W. Zimmermann, J. Fluid Mech., 2011, 673, 318-348.
20 C. Elphick, A. Hagberg and E. Meron, Phys. Rev. E: Stat. Phys., Plasmas, Fluids, Relat. Interdiscip. Top., 1999, 59, 5285.

21 A. L. Lin, A. Hagberg, A. Ardelea, M. Bertram, H. L. Swinney and E. Meron, Phys. Rev. E: Stat. Phys., Plasmas, Fluids, Relat. Interdiscip. Top., 2000, 62, 3790-3798.

22 L. Korzinov, M. Rabinovich and L. Tsimring, Phys. Rev. A: At., Mol., Opt. Phys., 1992, 46, 7601.

23 P. Coullet, J. Lega, B. Houchmanzadeh and J. Lajzerowicz, Phys. Rev. Lett., 1990, 65, 1352.

24 C. Elphick, A. Hagberg, B. Malomed and E. Meron, Phys. Lett. A, 1997, 230, 33-37.

25 I. Pérez-Arjona, F. Silva, G. J. de Valcárcel, E. Roldán and V. J. Sánchez-Morcillo, J. Opt. B: Quantum Semiclassical Opt., 2004, 6, S361.

26 V. J. Sánchez-Morcillo, V. Espinosa, I. Pérez-Arjona, F. Silva, G. J. de Valcárcel and E. Roldán, Phys. Rev. E: Stat., Nonlinear, Soft Matter Phys., 2005, 71, 066209.

27 I. Lengyel and I. R. Epstein, Science, 1991, 251, 650-652.

28 Handbook of Preparative Inorganic Chemistry, ed. G. Brauer, Academic, New York, 2nd edn, 1963.

29 A. P. Muñuzuri, M. Dolnik, A. M. Zhabotinsky and I. R. Epstein, J. Am. Chem. Soc., 1999, 121, 8065-8069.

30 L. Haim, PhD thesis, Ben-Gurion University of the Negev, 2014.

31 R. Manor, A. Hagberg and E. Meron, Europhys. Lett., 2008, 83, 10005.

32 A. Esteban-Martín, V. B. Taranenko, J. García, G. J. de Valcárcel and E. Roldán, Phys. Rev. Lett., 2005, 94, 223903.

33 V. B. Taranenko, A. Esteban-Martín, G. J. de Valcárcel and E. Roldán, Phys. Rev. E: Stat., Nonlinear, Soft Matter Phys., 2006, 73, 027201.

34 A. Hagberg and E. Meron, Phys. Rev. Lett., 1997, 78, 1166-1169.

35 A. Lampert and E. Meron, EPL, 2007, 78, 14002.

36 Y. Mau, A. Hagberg and E. Meron, Phys. Rev. E: Stat., Nonlinear, Soft Matter Phys., 2009, 80, 065203(R). 